\title{
A survey of cluster validity indices for automatic data clustering using differential evolution
}

\author{
SUPPLEMENTARY MATERIAL
}

\author{
Adán José-García \\ Department of Computer Science, CRIStAL Lab, \\ University of Lille \\ adan.jose@cinvestav.mx
}

\begin{abstract}
ACM Reference Format:
Adán José-García and Wilfrido Gómez-Flores. 2021. A survey of cluster validity indices for automatic data clustering using differential evolution: SUPPLEMENTARY MATERIAL. In 2021 Genetic and Evolutionary Computation Conference (GECCO '21), July 10-14, 2021, Lille, France. ACM, New York, NY, USA, 3 pages. https://doi.org/10.1145/3449639.3459341
\end{abstract}

\section{CLUSTER VALIDITY INDICES}

This section describes several cluster validity indices (CVIs), which have been used specifically as fitness functions in metaheuristic clustering algorithms to address the automatic clustering problem.

\subsection{Definitions}

Some basic definitions are given before presenting the CVIs:

- A pattern (or object) $\mathbf{x}$ is a single data item represented by a vector of measurements $\mathbf{x}=\left[x_{1}, x_{2}, \ldots, x_{D}\right]^{\top}$, where $x_{i} \in \mathbb{R}$ is a feature (or attribute) and D denotes the dimensionality.

- A dataset is denoted by $\mathbf{X}=\left\{\mathbf{x}_{1}, \mathbf{x}_{2}, \ldots, \mathbf{x}_{\mathrm{N}}\right\} \in \mathbb{R}^{\mathrm{D}}$, where $\mathrm{N}$ is the total number of patterns in the dataset.

- A clustering, denoted by $\mathbf{C}=\left\{\mathbf{c}_{\mathrm{k}} \mid \mathrm{k}=1, \ldots, \mathrm{K}\right\}$, refers to a set of mutually disjoint clusters that partition $\mathbf{X}$ into $\mathrm{K}$ groups.

- The number of objects in a cluster $\mathbf{c}_{\mathrm{k}}$ is denoted by $\mathrm{n}_{\mathrm{k}}=\left|\mathbf{c}_{\mathrm{k}}\right|$.

- The centroid of a cluster (or prototype) is expressed as $\overline{\mathbf{c}}_{\mathrm{k}}=$ $1 / n_{k} \mathbf{x}_{i} \in \mathbf{c}_{k} \mathbf{x}_{i}$, whereas the centroid of a dataset $\mathbf{X}$ is denoted by $\overline{\mathbf{X}}=1 / \mathrm{N}_{\mathbf{x}_{i} \in \mathbf{X}} \mathbf{x}_{i}$.

- A distance measure is a metric (or quasi-metric) used to quantify the proximity between two patterns. Probably the most common distance metric is the Euclidean distance, expressed as $\mathrm{d}_{\mathrm{e}}(\mathbf{x}, \mathbf{y})=$ $\sqrt{i=1}\left(x_{i}-y_{i}\right)^{2}$.

- The point symmetry distance [4] between the object $\mathbf{x}_{i}$ and the cluster $\mathbf{c}_{\mathrm{k}}$ is defined by

$$
\mathrm{d}_{\mathrm{ps}}\left(\mathbf{x}_{\mathrm{i}}, \mathbf{c}_{\mathrm{k}}\right)=\frac{1}{\mathrm{k}_{\mathrm{n}}} \min _{\mathbf{x}_{\mathrm{j}} \in \mathbf{c}_{\mathrm{k}}}\left(\mathrm{k}_{\mathrm{n}}\right)\left\{\mathrm{d}_{\mathrm{e}}\left(2 \overline{\mathbf{c}}_{\mathrm{k}}-\mathbf{x}_{\mathrm{i}}, \mathbf{x}_{\mathrm{j}}\right)\right\} .
$$

The point $2 \overline{\mathbf{c}}_{k}-\mathbf{x}_{i}$ is called the symmetrical (reflected) point of $\mathbf{x}_{i}$ with respect to the centroid of $\mathbf{c}_{k}$ and $k_{n}=2$ are its unique nearest neighbors. The function min can be seen as an adaptation

Permission to make digital or hard copies of all or part of this work for personal or classroom use is granted without fee provided that copies are not made or distributed for profit or commercial advantage and that copies bear this notice and the full citation on the first page. Copyrights for components of this work owned by others than ACM must be honored. Abstracting with credit is permitted. To copy otherwise, or republish, to post on servers or to redistribute to lists, requires prior specific permission and/or a fee. Request permissions from permissions@acm.org.

GECCO '21, July 10-14, 2021, Lille, France

(C) 2021 Association for Computing Machinery.

ACM ISBN 978-1-4503-8350-9/21/07 . \$ \$15.00

https://doi.org/10.1145/3449639.3459341

\author{
Wilfrido Gómez-Flores \\ Centro de Investigación y de Estudios Avanzados del IPN, \\ Unidad Tamaulipas \\ wgomez@cinvestav.mx
}

of the min function, where $\min (n)$ computes the sum of the $n$ lowest arguments.

\subsection{Cluster validity indices}

A CVI defines a relation between intracluster cohesion (withingroup scatter) and intercluster separation (between-group scatter) to estimate the quality of a clustering solution [25]. The 22 CVIs evaluated in this study are described in the next. An acronym is defined to identify each CVI, followed by an up arrow ( $\uparrow$ ) or a down arrow $(\downarrow)$ to indicate whether the index is to be maximized or minimized. A general description is provided for every CVI in terms of criteria of cluster cohesion and separation.

- The Dunn index $\left(\mathbf{D I}_{\uparrow}\right)$ [14]. This is a ratio-type index in which the cohesion is quantified by the maximum cluster diameter and the separation by the nearest neighbor distance. It is defined by

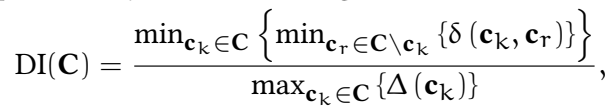

where

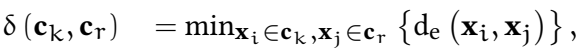

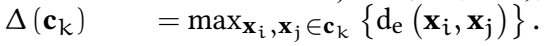

- The Calinski-Harabasz index $\left(\mathbf{C H}_{\uparrow}\right)$ [8]. This is a ratio-type index in which the cohesion is quantified by the sum of the distances of the patterns to their respective centroids, and the separation is measured by the sum of the distances from the centroids to the global prototype. It is defined by

$$
\mathrm{CH}(\mathbf{C})=\frac{\mathrm{N}-\mathrm{K}}{\mathrm{K}-1} \times \frac{\mathbf{c}_{\mathrm{k}} \in \mathbf{C} \mathrm{n}_{\mathrm{k}} \mathrm{d}_{\mathrm{e}}^{2}\left(\overline{\mathbf{c}}_{\mathrm{k}}, \overline{\mathbf{x}}\right)}{\mathbf{c}_{\mathrm{k}} \in \mathbf{C} \mathbf{x}_{\mathrm{i}} \in \mathbf{c}_{\mathrm{k}} \mathrm{d}_{\mathrm{e}}^{2}\left(\mathbf{x}_{\mathrm{i}}, \overline{\mathbf{c}}_{\mathrm{k}}\right)} .
$$

- The C-Index ( $\left.\mathbf{C I}_{\downarrow}\right)$ [22]. This is a normalized index in which the cohesion is quantified by the sum of the distances between all the patterns in the same cluster, and the separation is based on the sum of the smallest and largest distances between all the patterns in the dataset. It is computed by

$$
\mathrm{CI}(\mathbf{C})=\frac{\mathrm{S}(\mathbf{C})-\mathrm{S}_{\min }(\mathbf{C})}{\mathrm{S}_{\max }(\mathbf{C})-\mathrm{S}_{\min }(\mathbf{C})}
$$

where

$$
\begin{aligned}
& \mathrm{S}(\mathbf{C}) \quad=\mathbf{c}_{\mathrm{k}} \in \mathbf{C} \mathbf{x}_{\mathrm{i}}, \mathbf{x}_{\mathrm{j}} \in \mathbf{c}_{\mathrm{k}} \mathrm{d}_{\mathrm{e}}\left(\mathbf{x}_{\mathrm{i}}, \mathbf{x}_{\mathrm{j}}\right), \\
& \mathrm{S}_{\min }(\mathbf{C})=\min \left(\mathrm{n}_{w}\right)_{\mathbf{x}_{\mathrm{i}}, \mathbf{x}_{\mathrm{j}} \in \mathbf{X}}\left\{\mathrm{d}_{\mathrm{e}}\left(\mathbf{x}_{\mathrm{i}}, \mathbf{x}_{\mathrm{j}}\right)\right\} \text {, } \\
& \mathrm{S}_{\max }(\mathbf{C})=\max \left(\mathrm{n}_{w}\right)_{\mathbf{x}_{i}, \mathbf{x}_{j} \in \mathbf{X}}\left\{\mathrm{d}_{\mathrm{e}}\left(\mathbf{x}_{\mathrm{i}}, \mathbf{x}_{\mathbf{j}}\right)\right\} \text {. }
\end{aligned}
$$

and where $n_{w}$ is the number of pairs of objects in a partition that are in the same cluster: $n_{w}=\mathbf{c}_{k} \in \mathbf{C}\left(\begin{array}{c}n_{k} \\ 2\end{array}\right)$.

- The Davies-Bouldin index $\left(\mathbf{D B}_{\downarrow}\right)$ [11]. In this index, the cohesion is quantified by the mean distance of the objects to their 
respective centroids, and the separation quantifies the distance between centroids. It is expressed by

$$
\operatorname{DB}(\mathbf{C})=\frac{1}{\mathrm{~K}} \max _{\mathbf{c}_{\mathrm{k}} \in \mathbf{C}}\left\{\frac{\mathrm{S}\left(\mathbf{c}_{\mathrm{k}}\right)+\mathrm{S}\left(\mathbf{c}_{\mathrm{r}}\right)}{\mathrm{d}_{\mathrm{e}}\left(\overline{\mathbf{c}}_{\mathrm{k}}, \overline{\mathbf{c}}_{\mathrm{r}}\right)}\right\},
$$

where

$$
\mathrm{S}\left(\mathbf{c}_{\mathrm{k}}\right)=\frac{1}{\mathrm{n}_{\mathrm{k}}} \mathbf{x}_{\mathrm{i}} \in \mathbf{c}_{\mathrm{k}} \mathrm{d}_{\mathrm{e}}\left(\mathbf{x}_{\mathrm{i}}, \overline{\mathbf{c}}_{\mathrm{k}}\right) .
$$

- The Silhouette index $\left(\mathbf{S i l}_{\uparrow}\right)$ [30]. This index is a normalized summation-type index in which the cohesion is measured by the sum of the distances between all the points in the same cluster, and the separation is based on the nearest neighbor distance between points in different groups. It is defined by

$$
\operatorname{SI}(\mathbf{C})=\frac{1}{N} \mathbf{c}_{k} \in \mathbf{C} \mathbf{x}_{i} \in \mathbf{c}_{k} \frac{\mathrm{b}\left(\mathbf{x}_{i}, \mathbf{c}_{\mathrm{k}}\right)-\mathrm{a}\left(\mathbf{x}_{\mathrm{i}}, \mathbf{c}_{\mathrm{k}}\right)}{\max \left\{\mathrm{b}\left(\mathbf{x}_{i}, \mathbf{c}_{\mathrm{k}}\right), \mathrm{a}\left(\mathbf{x}_{\mathrm{i}}, \mathbf{c}_{\mathrm{k}}\right)\right\}},
$$

where

$$
\begin{aligned}
& \mathrm{a}\left(\mathbf{x}_{\mathrm{i}}, \mathbf{c}_{\mathrm{k}}\right)=\frac{1}{\mathrm{n}_{\mathrm{k}}-1} \mathbf{x}_{\mathrm{j}} \in \mathbf{c}_{\mathrm{k}} \mathrm{d}_{\mathrm{e}}\left(\mathbf{x}_{\mathrm{i}}, \mathbf{x}_{\mathrm{j}}\right), \\
& \mathrm{b}\left(\mathbf{x}_{\mathrm{i}}, \mathbf{c}_{\mathrm{k}}\right)=\min _{\mathbf{c}_{\mathrm{r}} \in \mathbf{C} \backslash \mathbf{c}_{\mathrm{k}}}\left\{\frac{1}{\mathrm{n}_{\mathrm{r}}} \mathbf{x}_{\mathrm{j}} \in \mathbf{c}_{\mathrm{r}} \mathrm{d}_{\mathrm{e}}\left(\mathbf{x}_{\mathrm{i}}, \mathbf{x}_{\mathrm{j}}\right)\right\} .
\end{aligned}
$$

- The Xie-Beni index $\left(\mathbf{X B}_{\downarrow}\right)$ [38]. This is the ratio of the total variation to the minimum separation of the clusters and is defined by

$$
\mathrm{XB}(\mathbf{C})=\frac{\mathbf{c}_{\mathrm{k}} \in \mathbf{C} \mathbf{x}_{\mathrm{i}} \in \mathbf{c}_{\mathrm{k}} \mathrm{d}_{\mathrm{e}}^{2}\left(\mathbf{x}_{\mathrm{i}}, \overline{\mathbf{c}}_{\mathrm{k}}\right)}{\mathrm{N} \min _{\mathbf{c}_{\mathrm{k}} \in \mathbf{C}} \min _{\mathbf{c}_{\mathrm{r}} \in \mathbf{C} \backslash \mathbf{c}_{\mathrm{k}}}\left\{\mathrm{d}_{\mathrm{e}}^{2}\left(\overline{\mathbf{c}}_{\mathrm{k}}, \overline{\mathbf{c}}_{\mathrm{r}}\right)\right\}} .
$$

- The generalized Dunn indices ${ }^{1}\left(\right.$ gD31 $_{\uparrow}$, gD41 $_{\uparrow}$, gD51 $_{\uparrow}$, gD33 $_{\uparrow}$, gD43 $\boldsymbol{H}_{\uparrow}$, and $\mathbf{g D 5 3} \uparrow$ ) [6]. These indices are modifications of the original Dunn index, and include a combination of three variations of $\delta$ (a separation criterion)

$\delta_{3}\left(\mathbf{c}_{\mathrm{k}}, \mathbf{c}_{\mathrm{r}}\right)=\frac{1}{n_{\mathrm{k}} \times n_{\mathrm{r}}} \mathbf{x}_{\mathrm{i}} \in \mathbf{c}_{\mathrm{k}}, \mathbf{x}_{\mathrm{j}} \in \mathbf{c}_{\mathrm{r}} \mathrm{d}_{\mathrm{e}}\left(\mathbf{x}_{\mathrm{i}}, \mathbf{x}_{\mathrm{j}}\right)$,

$\delta_{4}\left(\mathbf{c}_{\mathrm{k}}, \mathbf{c}_{\mathrm{r}}\right)=\mathrm{d}_{\mathrm{e}}\left(\overline{\mathbf{c}}_{\mathrm{k}}, \overline{\mathbf{c}}_{\mathrm{r}}\right)$,

$\delta_{5}\left(\mathbf{c}_{\mathrm{k}}, \mathbf{c}_{\mathrm{r}}\right)=\frac{1}{n_{\mathrm{k}}+\mathrm{n}_{\mathrm{r}}}\left[\mathbf{x}_{\mathrm{i}} \in \mathbf{c}_{\mathrm{k}} \mathrm{d}_{\mathrm{e}}\left(\mathbf{x}_{\mathrm{i}}, \overline{\mathbf{c}}_{\mathrm{k}}\right)+\mathbf{x}_{\mathrm{j}} \in \mathbf{c}_{\mathrm{r}} \mathrm{d}_{\mathrm{e}}\left(\mathbf{x}_{\mathrm{j}}, \overline{\mathbf{c}}_{\mathrm{r}}\right)\right]$

and two variations of $\Delta$ (a cohesion criterion)

$$
\begin{aligned}
& \Delta_{1}\left(\mathbf{c}_{\mathrm{k}}\right)=\max _{\mathbf{x}_{\mathrm{i}}, \mathbf{x}_{\mathrm{j}} \in \mathbf{c}_{\mathrm{k}}}\left\{\mathrm{d}_{\mathrm{e}}\left(\mathbf{x}_{\mathrm{i}}, \mathbf{x}_{\mathrm{j}}\right)\right\}, \\
& \Delta_{3}\left(\mathbf{c}_{\mathrm{k}}\right)=\frac{2}{\mathrm{n}_{\mathrm{k}}} \mathbf{x}_{\mathrm{i}} \in \mathbf{c}_{\mathrm{k}} \mathrm{d}_{\mathrm{e}}\left(\mathbf{x}_{\mathrm{i}}, \overline{\mathbf{c}}_{\mathrm{k}}\right) .
\end{aligned}
$$

- The S_Dbw index $\left(\mathbf{S D b w}_{\downarrow}\right)$ [18]. This is a ratio-type index that is based on the Euclidean norm $\|\mathbf{x}\|=\left(\mathbf{x}^{\top} \mathbf{x}\right)^{1 / 2}$, the standard deviation of the dataset $\sigma(\mathbf{X})=1 /|\mathbf{X}|_{\mathbf{x}_{i} \in \mathbf{X}}\left(\mathbf{x}_{i}-\overline{\mathbf{X}}\right)^{2}$, and the standard deviation of the partition $\operatorname{stdev}(\mathbf{C})=1 / K \sqrt{\mathbf{c}_{\mathrm{k}} \in \mathbf{C}\left\|\sigma\left(\mathbf{c}_{\mathrm{k}}\right)\right\|}$. It is defined by

$$
\begin{array}{ll}
\operatorname{Scat}(\mathbf{C}) & =\frac{1}{\mathrm{~K}} \mathbf{c}_{\mathrm{k}} \in \mathbf{C} \frac{\left\|\sigma\left(\mathbf{c}_{\mathrm{k}}\right)\right\|}{\|\sigma(\mathbf{X})\|}, \\
\operatorname{Dbw}(\mathbf{C}) & =\frac{1}{\mathrm{~K}(\mathrm{~K}-1)} \mathbf{c}_{\mathrm{k}} \in \mathbf{C} \mathbf{c}_{\mathrm{r}} \in \mathbf{C} \backslash \mathbf{c}_{\mathrm{k}} \frac{\operatorname{den}\left(\mathbf{c}_{\mathrm{k}}, \mathbf{c}_{\mathrm{r}}\right)}{\max \left\{\operatorname{den}\left(\mathbf{c}_{\mathrm{k}}\right), \operatorname{den}\left(\mathbf{c}_{\mathrm{r}}\right)\right\}}, \\
\operatorname{SDbw}(\mathbf{C}) & =\operatorname{Scat}(\mathbf{C})+\operatorname{Dbw}(\mathbf{C}),
\end{array}
$$

where

${ }^{1}$ Bedeck and Pal proposed 18 variants, but we have selected those that were analyzed by Arbelaitz et al. [2], since these showed the best results.

$$
\begin{aligned}
& \operatorname{den}\left(\mathbf{c}_{\mathrm{k}}\right) \quad={ }_{\mathbf{x}_{i} \in \mathbf{c}_{\mathrm{k}}} \mathrm{f}\left(\mathbf{x}_{\mathrm{i}}, \overline{\mathbf{c}}_{\mathrm{k}}\right), \\
& \operatorname{den}\left(\mathbf{c}_{k}, \mathbf{c}_{r}\right)=\mathbf{x}_{i} \in \mathbf{c}_{k} \cup \mathbf{c}_{r} \mathrm{f}\left(\mathbf{x}_{i}, \frac{\overline{\mathbf{c}}_{k}+\overline{\mathbf{c}}_{\mathrm{r}}}{2}\right),
\end{aligned}
$$

and

$$
\mathrm{f}\left(\mathbf{x}_{\mathrm{i}}, \overline{\mathbf{c}}_{\mathrm{k}}\right)=\left\{\begin{array}{ll}
0 & \text { if } \mathrm{d}_{\mathrm{e}}\left(\mathbf{x}_{\mathrm{i}}, \overline{\mathbf{c}}_{\mathrm{k}}\right)>\operatorname{stdev}(\mathbf{C}) . \\
1 & \text { otherwise }
\end{array} .\right.
$$

- The CS index $\left(\mathbf{C S}_{\downarrow}\right)$ [9]. This is a ratio-type index that quantifies the cohesion by using the cluster diameters. The measure of separation is based on the nearest neighbor distance between prototypes. It is computed by

$$
\mathrm{CS}(\mathbf{C})=\frac{\mathbf{c}_{\mathrm{k}} \in \mathbf{C} \Delta\left(\mathbf{c}_{\mathrm{k}}\right)}{\mathbf{c}_{\mathrm{k}} \in \mathbf{C} \min _{\mathbf{c}_{\mathrm{r}} \in \mathbf{C} \backslash \mathbf{c}_{\mathrm{k}}}\left\{\mathrm{d}_{\mathrm{e}}\left(\overline{\mathbf{c}}_{\mathrm{k}}, \overline{\mathbf{c}}_{\mathrm{r}}\right)\right\}},
$$

where

$$
\Delta\left(\mathbf{c}_{\mathrm{k}}\right)=\frac{1}{\mathrm{n}_{\mathrm{k}}} \max _{\mathbf{x}_{\mathrm{i}} \in \mathbf{c}_{\mathrm{k}}} \max _{\mathbf{j}} \in \mathrm{c}_{\mathrm{k}}\left(\mathrm{d}_{\mathrm{e}}\left(\mathbf{x}_{\mathrm{i}}, \mathbf{x}_{\mathrm{j}}\right)\right\} .
$$

- The Modified Davies-Bouldin index $\left(\mathbf{m D B}_{\downarrow}\right)$ [25]. This variation of the DB index estimates the separation by the sum of the minimum distances between prototypes. It is defined by

$$
\operatorname{mDB}(\mathbf{C})=\frac{1}{\mathrm{~K}_{\mathbf{c}_{\mathrm{k}} \in \mathbf{C}}} \frac{\max _{\mathbf{c}_{\mathrm{r}} \in \mathbf{C} \backslash \mathbf{c}_{\mathrm{k}}}\left\{\mathrm{S}\left(\mathbf{c}_{\mathrm{k}}\right)+\mathrm{S}\left(\mathbf{c}_{\mathrm{r}}\right)\right\}}{\min _{\mathbf{c}_{\mathrm{r}} \in \mathbf{C} \backslash \mathbf{c}_{\mathrm{k}}}\left\{\mathrm{d}_{\mathrm{e}}\left(\overline{\mathbf{c}}_{\mathrm{k}}, \overline{\mathbf{c}}_{\mathrm{r}}\right)\right\}} .
$$

- The Score Function index $\left(\mathbf{S F}_{\uparrow}\right)$ [33]. This is a summation-type index in which the separation is measured by the sum of the distances from each centroid to the global prototype, and the cohesion is quantified by the mean distance of the objects to their respective centroids. It is computed by

$$
\operatorname{SF}(\mathbf{C})=1-\frac{1}{\mathrm{e}^{\mathrm{e}}}
$$

where

$$
\begin{aligned}
\operatorname{wcd}(\mathbf{C}) & =\mathbf{c}_{\mathrm{k} \in \mathbf{C}} \frac{1}{\mathrm{n}_{\mathrm{k}}} \mathbf{x}_{\mathrm{i}} \in \mathbf{c}_{\mathrm{k}} \mathrm{d}_{\mathrm{e}}\left(\mathbf{x}_{\mathrm{i}}, \overline{\mathbf{c}}_{\mathrm{k}}\right), \\
\operatorname{bcd}(\mathbf{C}) & =\frac{\mathbf{c}_{\mathrm{k}} \in \mathbf{C} \mathrm{n}_{\mathrm{k}} \mathrm{d}_{\mathrm{e}}\left(\overline{\mathbf{c}}_{\mathrm{k}}, \overline{\mathbf{X}}\right)}{\mathrm{N} \times \mathrm{K}}
\end{aligned}
$$

- The PBM index $(\mathbf{P B M} \uparrow)$ [26]. This index is defined as the product of cohesion and separation measures. The former is the sum of all pattern distances in a cluster to their respective centroids, whereas the latter is quantified by the maximum distance between the centroids. The PBM index is given by

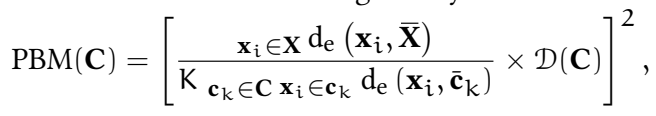

where

$$
\mathcal{D}(\mathbf{C})=\max _{\mathbf{c}_{\mathrm{k}}, \mathbf{c}_{\mathrm{r}} \in \mathbf{C}}\left\{\mathrm{d}_{\mathrm{e}}\left(\overline{\mathbf{c}}_{\mathrm{k}}, \overline{\mathbf{c}}_{\mathrm{r}}\right)\right\} .
$$

- The Symmetry index $\left(\mathbf{S y m}_{\uparrow}\right)$ [4]. This ratio-type index is an adaptation of the PBM index [26]: the cohesion is quantified by the sum of the point symmetry distances in the same cluster, and the separation is quantified by the maximum Euclidean distance between the centroids. This index is defined by

$$
\operatorname{Sym}(\mathbf{C})=\frac{\max _{\mathbf{c}_{k}, \mathbf{c}_{\mathrm{r}} \in \mathbf{C}}\left\{\mathrm{d}_{\mathrm{e}}\left(\overline{\mathbf{c}}_{\mathrm{k}}, \overline{\mathbf{c}}_{\mathrm{r}}\right)\right\}}{\mathrm{K}_{\mathbf{c}_{\mathrm{k}} \in \mathbf{C} \mathbf{x}_{\mathrm{i}} \in \mathbf{c}_{\mathrm{k}}} \mathrm{d}_{\mathrm{ps}}\left(\mathbf{x}_{\mathrm{i}}, \mathbf{c}_{\mathrm{k}}\right)}
$$

- Point Symmetry-Distance indices ${ }^{2}\left(\mathbf{S D B}_{\downarrow}\right.$ and $\left.\mathbf{S D I}_{\uparrow}\right)$ [31]. These are based on the PS distance and modify the cohesion criterion of the Davies-Bouldin and Dunn indices. The SDB index is

${ }^{2}$ Saha and Bandyopadhyay presented nine indices but we have selected the two most representative. 
computed the same way as the DB, but the computation of $\mathrm{S}$ is refined as follows:

$$
\mathrm{S}\left(\mathbf{c}_{\mathrm{k}}\right)=\frac{1}{\mathrm{n}_{\mathrm{k}}} \mathbf{x}_{\mathrm{i}} \in \mathbf{c}_{\mathrm{k}} \mathrm{d}_{\mathrm{ps}}\left(\mathbf{x}_{\mathrm{i}}, \overline{\mathbf{c}}_{\mathrm{k}}\right) .
$$

On the other hand, SDI is a modification of DI, where the cohesion criterion $\Delta$ is defined by

$$
\Delta\left(\mathbf{c}_{\mathrm{k}}\right)=\max _{\mathbf{x}_{\mathrm{i}} \in \mathbf{c}_{\mathrm{k}}}\left\{\mathrm{d}_{\mathrm{ps}}\left(\mathbf{x}_{\mathrm{i}}, \overline{\mathbf{c}}_{\mathrm{k}}\right)\right\} .
$$

- The COP index $\left(\mathbf{C O P}_{\downarrow}\right)$ [16]. This is a ratio-type index in which the cohesion is quantified by the sum of the distances of the patterns to their respective centroids, and the separation is based on the furthest neighbor distance between patterns. It is defined by

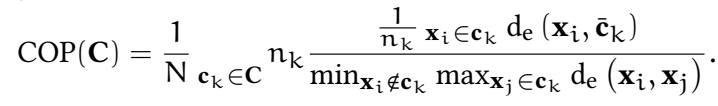

- The SV index $\left(\mathbf{S V}_{\uparrow}\right)$ [42]. In this ratio-type index, the measure of cohesion is based on the distances from the border patterns to their respective centroids, and the measure of separation is based on the nearest neighbor distance between prototypes. It is computed by

$$
\mathrm{SV}(\mathbf{C})=\frac{\mathbf{c}_{\mathrm{k}} \in \mathbf{C} \min _{\mathbf{c}_{\mathrm{r}} \in \mathbf{C} \backslash \mathbf{c}_{\mathrm{k}}}\left\{\mathrm{d}_{\mathrm{e}}\left(\overline{\mathbf{c}}_{\mathrm{k}}, \overline{\mathbf{c}}_{\mathrm{r}}\right)\right\}}{\mathbf{c}_{\mathrm{k}} \in \mathbf{C}\left(\frac{10}{n_{\mathrm{k}}}\right) \max _{\mathbf{x}_{\mathrm{i}} \in \mathbf{c}_{\mathrm{k}}}\left(\frac{\mathrm{n}_{\mathrm{k}}}{10}\right)\left\{\mathrm{d}_{\mathrm{e}}\left(\mathbf{x}_{\mathrm{i}}, \overline{\mathbf{c}}_{\mathrm{k}}\right)\right\}} .
$$

\section{REFERENCES}

[1] Ultsch Alfred. 2005. Clustering with SOM: $\mathrm{U}^{*} \mathrm{C}$. In Proceedings Workshop on Self-Organizing Maps (WSOM). Paris, France, 75-82.

[2] Olatz Arbelaitz, Ibai Gurrutxaga, Javier Muguerza, Jesús M. Pérez, and Iñigo Perona. 2013. An Extensive Comparative Study of Cluster Validity Indices. Pattern Recognition 46, 1 (2013), 243-256.

[3] Sanghamitra Bandyopadhyay and Ujjwal Maulik. 2002. Genetic Clustering for Automatic Evolution of Clusters and Application to Image Classification. Pattern Recognition 35, 6 (2002), 1197-1208.

[4] Sanghamitra Bandyopadhyay and Sriparna Saha. 2008. A Point Symmetry-Based Clustering Technique for Automatic Evolution of Clusters. IEEE Transactions on Knowledge and Data Engineering 20, 11 (2008), 1441-1457.

[5] J. C. Bezdek, W. Q. Li, Y. Attikiouzel, and M. Windham. 1997. A Geometric Approach to Cluster Validity for Normal Mixtures. Soft Computing - A Fusion of Foundations, Methodologies and Applications 1, 4 (1997), 166-179.

[6] James C. Bezdek and N. R. Pal. 1998. Some New Indexes of Cluster Validity. IEEE Transactions on Systems, Man, and Cybernetics, Part B (Cybernetics) 28, 3 (1998), 301-15.

[7] Marcel Brun, Chao Sima, Jianping Hua, James Lowey, Brent Carroll, Edward Suh, and Edward R. Dougherty. 2007. Model-based Evaluation of Clustering Validation Measures. Pattern Recognition 40, 3 (2007), 807-824.

[8] T. Calinski and J. Harabasz. 1974. A Dendrite Method for Cluster Analysis. Communications in Statistics - Theory and Methods 3, 1 (1974), 1-27.

[9] Chien Hsing Chou, M. C. Su, and E. Lai. 2004. A New Cluster Validity Measure and its Application to Image Compression. Pattern Analysis and Applications 7, 2 (2004), 205-220.

[10] Swagatam Das, Ajith Abraham, and Amit Konar. 2008. Automatic Clustering Using an Improved Differential Evolution Algorithm. IEEE Transactions on Systems, Man and Cybernetics 38, 1 (2008), 218-237.

[11] David L. Davies and Donald W. Bouldin. 1979. A Cluster Separation Measure. IEEE Transactions on Pattern Analysis and Machine Intelligence 1, 2 (1979), 224-227.

[12] Evgenia Dimitriadou, Sara Dolničar, and Andreas Weingessel. 2002. An Examination of Indexes for Determining the Number of Clusters in Binary Data Sets. Psychometrika 67, 1 (2002), 137-159.

[13] Dheeru Dua and Casey Graff. 2017. UCI Machine Learning Repository. http: //archive.ics.uci.edu/ml

[14] J. C. Dunn. 1973. A Fuzzy Relative of the ISODATA Process and Its Use in Detecting Compact Well-Separated Clusters. Fournal of Cybernetics 3, 3 (1973), 32-57.

[15] Emanuel Falkenauer. 1998. Genetic Algorithms and Grouping Problems. John Wiley and Sons Ltd, Chichester, England. 238 pages.

[16] Ibai Gurrutxaga, Iñaki Albisua, Olatz Arbelaitz, José I. Martín, Javier Muguerza, Jesús M. Pérez, and Iñigo Perona. 2010. SEP/COP: An Efficient Method to Find the Best Partition in Hierarchical Clustering Based on a New Cluster Validity Index. Pattern Recognition 43, 10 (2010), 3364-3373.
[17] Ibai Gurrutxaga, Javier Muguerza, Olatz Arbelaitz, Jesús M. Pérez, and José I. Martín. 2011. Towards a Standard Methodology to Evaluate Internal Cluster Validity Indices. Pattern Recognition Letters 32, 3 (2011), 505-515.

[18] M. Halkidi and M. Vazirgiannis. 2001. Clustering Validity Assessment: Finding the Optimal Partitioning of a Data Set. In Proceedings 2001 IEEE International Conference on Data Mining. IEEE, California, USA, 187-194.

[19] Julia Handl and Joshua Knowles. 2007. An Evolutionary Approach to Multiobjective Clustering. IEEE Transactions on Evolutionary Computation 11, 1 (2007), 56-76.

[20] Hong He and Yonghong Tan. 2012. A Two-Stage Genetic Algorithm for Automatic Clustering. Neurocomputing 81 (2012), 49-59.

[21] Lawrence Hubert and Phipps Arabie. 1985. Comparing Partitions. Fournal of Classification 2, 1 (1985), 193-218.

[22] L. J. Hubert and J. R. Levin. 1976. Ageneral Statistical Framework for Assessing Categorical Clustering in Free Recall. Psychological Bulleting 83 (1976), 10721080.

[23] Adán José-García and Wilfrido Gómez-Flores. 2016. Automatic Clustering Using Nature-Inspired Metaheuristics: A Survey. Applied Soft Computing 41 (2016), 192-213.

[24] Adán José-García and Wilfrido Gómez-Flores. 2017. Evolutionary Clustering Using Multi-prototype Representation and Connectivity Criterion. In 9th Mexican Conference on Pattern Recognition.

[25] Minho Kim and R. S. Ramakrishna. 2005. New Indices for Cluster Validity Assessment. Pattern Recognition Letters 26, 15 (2005), 2353-2363.

[26] Ujjwal Maulik and Sanghamitra Bandyopadhyay. 2002. Performance Evaluation of Some Clustering Algorithms and Validity Indices. IEEE Transactions on Pattern Analysis and Machine Intelligence 24, 12 (2002), 1650-1654.

[27] Glenn W. Milligan and Martha C. Cooper. 1985. An Examination of Procedures for Determining the Number of Clusters in a Data Set. Psychometrika 50, 2 (1985), $159-179$.

[28] Satyasai Jagannath Nanda and Ganapati Panda. 2014. A Survey on Nature Inspired Metaheuristic Algorithms for Partitional Clustering. Swarm and Evolutionary Computation 16 (2014), 1-18.

[29] Mahamed Omran, Ayed Salman, and Andries P. Engelbrecht. 2005. Dynamic Clustering using Particle Swarm Optimization with Application in Image Segmentation. Pattern Analysis and Applications 8, 4 (2005), 332-344.

[30] Peter J. Rousseeuw. 1987. Silhouettes: A Graphical Aid to the Interpretation and Validation of Cluster Analysis. F. Comput. Appl. Math. 20 (1987), 53-65.

[31] Sriparna Saha and Sanghamitra Bandyopadhyay. 2009. Performance Evaluation of Some Symmetry-Based Cluster Validity Indexes. IEEE Transactions on Systems, Man, and Cybernetics, Part C (Applications and Reviews) 39, 4 (2009), 420-425.

[32] Sriparna Saha and Sanghamitra Bandyopadhyay. 2012. Some Connectivity Based Cluster Validity Indices. Applied Soft Computing 12, 5 (2012), 1555-1565.

[33] Sandro Saitta, Benny Raphael, and Ian F. C. Smith. 2007. A Bounded Index for Cluster Validity. In Machine Learning and Data Mining in Pattern Recognition. Springer Berlin Heidelberg, Berlin, Heidelberg, 174-187.

[34] Rainer Storn and Kenneth Price. 1997. Differential Evolution - A Simple and Efficient Heuristic for Global Optimization Over Continuous Spaces. Fournal of Global Optimization 11, 4 (1997), 341-359.

[35] El Ghazali Talbi. 2009. Metaheuristics from Design to Implementation. John Wiley and Sons. 1-3 pages.

[36] Sergios Theodoridis and Konstantinos Koutrumbas. 2009. Pattern Recognition (fourth ed.). Elsevier Inc.

[37] CJ Willmott and K Matsuura. 2005. Advantages of the Mean Absolute Error (MAE) over the Root Mean Square Error (RMSE) in Assessing Average Model Performance. Climate Research 30 (2005), 79-82.

[38] Xuanli Lisa Xie and Gerardo Beni. 1991. A Validity Measure for Fuzzy Clustering. IEEE Transactions on Pattern Analysis and Machine Intelligence 13, 8 (1991), 841847

[39] Rui Xu, Jie Xu, and Donald C Wunsch. 2012. A Comparison Study of Validity Indices on Swarm-Intelligence-Based Clustering. IEEE Transactions on Systems, Man, and Cybernetics, Part B (Cybernetics) 42, 2 (2012).

[40] Yanchi Liu, Zhongmou Li, Hui Xiong, Xuedong Gao, Junjie Wu, and Sen Wu. 2013. Understanding and Enhancement of Internal Clustering Validation Measures. IEEE Transactions on Cybernetics 43, 3 (2013), 982-994.

[41] Jian-Hua Yeh, Fei-Jie Joung, and Jia-Chi Lin. 2014. CDV Index: A Validity Index for Better Clustering Quality Measurement. Journal of Computer and Communications 02, 04 (2014), 163-171.

[42] Krista Rizman Žalik and Borut Žalik. 2011. Validity index for clusters of different sizes and densities. Pattern Recognition Letters 32, 2 (2011), 221-234.

[43] Wen-Jun Zhang and Xiao-Feng Xie. 2003. DEPSO: Hybrid Particle Swarm with Differential Evolution Operator. In IEEE International Conference on Systems, Man and Cybernetics., Vol. 4. IEEE, 3816-3821. 